\title{
Le jeu dans tous ses espaces
}

\section{Hovig Ter Minassian, Samuel Rufat et Manouk Borzakian}

\section{(2) OpenEdition}

\section{Journals}

\section{Édition électronique}

URL : https://journals.openedition.org/sdj/822

DOI : $10.4000 /$ sdi. 822

ISSN : 2269-2657

\section{Éditeur}

Laboratoire EXPERICE - Centre de Recherche Interuniversitaire Expérience Ressources Culturelles Education

\section{Référence électronique}

Hovig Ter Minassian, Samuel Rufat et Manouk Borzakian, «Le jeu dans tous ses espaces », Sciences du jeu [En ligne], 8 | 2017, mis en ligne le 26 décembre 2017, consulté le 21 septembre 2021. URL : http://journals.openedition.org/sdj/822 ; DOI : https://doi.org/10.4000/sdj.822

Ce document a été généré automatiquement le 21 septembre 2021.

\section{c) ()요}

La revue Sciences du jeu est mise à disposition selon les termes de la Licence Creative Commons Attribution - Pas d'Utilisation Commerciale - Pas de Modification 4.0 International. 


\title{
Le jeu dans tous ses espaces
}

\author{
Hovig Ter Minassian, Samuel Rufat et Manouk Borzakian
}

1 Dans l'étude des jeux, les travaux pionniers ont longtemps conforté la discontinuité supposée entre les espaces et temps du jeu et ceux de la vie quotidienne. Le jeu serait ainsi une activité particulière se déroulant « dans certaines limites fixées de temps et de lieu ", et qui resterait indemne des dynamiques sociales, économiques, politiques ou géographiques de la «vie courante» (Huizinga, 1951, 51). Avec J. Huizinga (1951) et R. Callois (1967), les jeux ont accédé au statut de dimension fondamentale des sociétés, mais en leur accordant une place centrale, les sciences sociales ont quelque peu délaissé leur spatialité. Au-delà des présupposés théoriques datés et de la conclusion commune faisant du jeu une sorte de deus ex machina de toute société, la cause est entendue : il y a du jeu, ou plutôt des jeux dans toutes les sociétés et comprendre celles-ci passe avantageusement par l'étude de ceux-là. Le jeu est à la fois une attitude, une pratique culturelle, une forme de socialisation, un objet matériel (parfois à fonction identitaire ou mémorielle), un produit artisanal ou industriel, une méthode d'acquisition de compétences, de la maternelle à la formation professionnelle. Si donc on joue (presque) à tout et (presque) partout, cette généralisation masque en fait une forte hétérogénéité à la fois des outils de production et de consommation, ainsi que des pratiques, du jeu de billes dans la cour de récréation aux tournois électroniques de la Dreamhack à Malmö, $\mathrm{du}$ plateau traditionnel en bois de l'awalé en Côte d'Ivoire aux superproductions vidéoludiques AAA qui prolongent (ou précèdent) les blockbusters du cinéma. Les jeux occupent une place variable selon les individus et les groupes sociaux, mais aussi selon les territoires, avec des usages, des socialisations et des enjeux différents en fonction des contextes de production et d'utilisation. Comme tout fait social, la pratique du jeu a une dimension spatiale. C'est à ces différentes spatialités du jeu que le $n^{\circ} 8$ de la revue Sciences du jeu est consacré.

2 Loin de toute posture disciplinaire, au-delà de la contribution de nombreux géographes, ce numéro invite à explorer l'intérêt heuristique d'entrer par les spatialités et les territoires pour éclairer le jeu, les joueurs, leurs pratiques et leurs représentations. Dans la perspective pluridisciplinaire de la revue, l'objectif est de faire de l'espace et des territoires une clé de lecture, pour montrer ce qu'apporte l'entrée par l'espace et les territoires du jeu à la compréhension des pratiques et phénomènes 
ludiques dans leur diversité (production, consommation, usages, cultures). Aujourd'hui, les jeux envahissent les espaces: de nouvelles formes de pratiques ludiques apparaissent (comme les jeux d'évasion récréatifs, ou escape rooms), les jeux de géolocalisation comme Pokémon Go rencontrent un succès planétaire et la nomadisation des supports permet d'embarquer son jeu préféré dans les transports, les salles de classe et jusque dans les toilettes. Pour appréhender ces transformations, nous proposons une représentation théorique de ces spatialités, de leurs articulations, à différentes échelles, de leurs productions par les pratiques, de leurs articulations aux sphères de la production et des univers culturels plus larges. Puis, nous essayons de montrer ce que ces spatialités ont à nous dire du jeu et des joueurs, en dégageant les principales perspectives de recherche.

\section{L'écosystème ludique}

Il nous semble difficile de produire une analyse in vitro des pratiques et des productions ludiques. L'entrée par l'espace et les territoires apporte la nécessaire contextualisation, en réinscrivant les pratiques, productions et représentations dans leur environnement et leurs dynamiques croisées, de façon à comprendre les logiques à l'œuvre et toute la diversité des phénomènes et des pratiques ludiques. Pour nous repérer dans ces différentes spatialités, nous proposons une représentation schématique de ce que nous suggérons d'appeler "l'écosystème ludique ». L'expression désigne l'association entre, d'une part, les acteurs interagissant entre eux et, d'autre part, les espaces dans lesquels ils se situent. Entre ces espaces et ces acteurs existent des flux (d'argent, de personnes, d'idées), qui contribuent à la dynamique d'ensemble de l'écosystème (croissance ou stagnation, inclusion ou exclusion, etc.) et déterminent son degré d'ouverture aux systèmes connexes (le monde de l'éducation ou du cinéma par exemple) ainsi qu'aux flux extérieurs (les politiques non spécifiquement dédiées au jeu, l'environnement économique, etc).

Pour caractériser cet écosystème, notre réflexion part d'une proposition théorique de modéliser trois dimensions spatiales du jeu vidéo : l'espace du joueur, l'espace dans le jeu et l'espace autour du jeu (Rufat, Ter Minassian, 2011). Nous l'avons étendue aux jeux en général, en cherchant à qualifier à la fois les espaces, territoires, et pratiques spatiales qu'ils suscitent. La schématisation que nous en faisons distingue la sphère de la production, la sphère de la consommation, et l'interface entre les deux où s'articulent les différents types d'espaces, de territoires et de réseaux qui forment le support des pratiques et phénomènes ludiques dans toute leur diversité (figure 1). 
Figure 1. Les dimensions spatiales du jeu

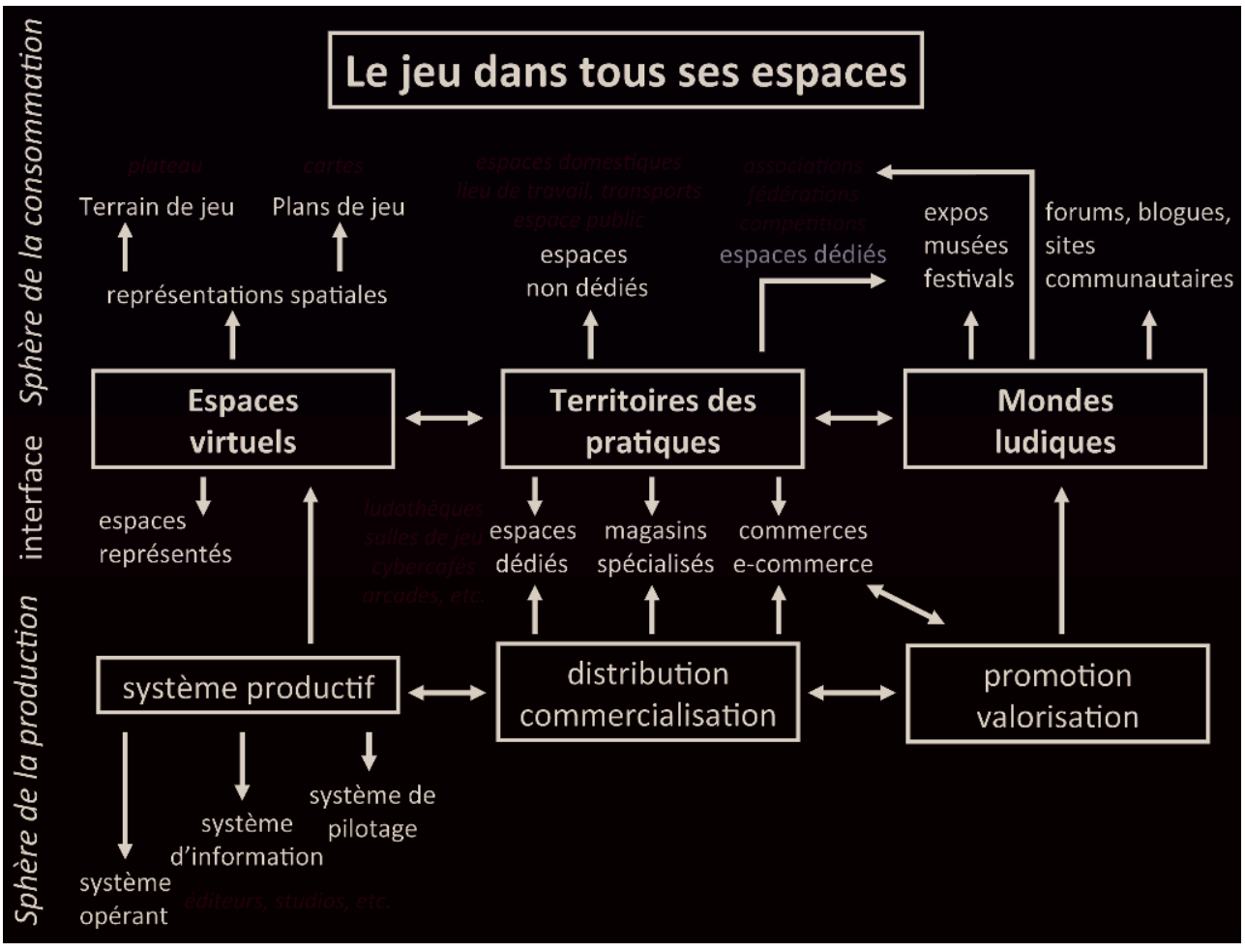

5 La sphère de la production regroupe l'ensemble des acteurs et des espaces qui, en amont des pratiques, concourent aux activités de conception, de financement, de production, de distribution et de commercialisation des jeux. Ce vaste ensemble constitué d'acteurs hétérogènes (publics et privés, grandes et petites entreprises, éditeurs et indépendants plus ou moins professionnalisés) est d'autant plus difficile à délimiter qu'il existe aujourd'hui de nombreuses interfaces au sein de l'écosystème des activités ludiques, entre sphère de la production et sphère de la consommation. Du côté de cette dernière, l'entrée par la dimension spatiale du jeu permet également d'analyser la différenciation territoriale des pratiques ludiques. Une conception dominante $\mathrm{du}$ "territoire " en géographie invite à le considérer comme un espace approprié et aménagé par un groupe social. Dans le cas des jeux, du backgammon en Turquie au rugby en Grande-Bretagne ou dans le sud-ouest de la France, en passant par les jeux de rôle grandeur nature dans le nord de l'Europe et le go en Asie de l'Est, il serait aisé de circonscrire certaines productions, pratiques, ou modalités de la pratique à des aires régionales ou nationales, même si bien souvent, les éléments de différenciation et de diffusion de ces pratiques sont peu documentés.

\section{Des espaces de la production aux espaces de la consommation}

6 Dans l'étude de l'industrie du jeu, l'entrée par l'espace permet de comprendre comment s'opère la territorialisation de la production. Dans le cas du jeu vidéo en Australie, J. Banks et S. Cunningham (2016) s'intéressent par exemple à la polarisation des activités de production de jeux vidéo dans les principales métropoles du pays (Melbourne, Brisbane et Sidney), mais surtout aux différences de types de contenu d'une 
communauté à l'autre. Ils formulent l'hypothèse que les concepteurs de jeu (game designers) de Sydney ont tendance à privilégier le commerce interentreprises (businessto-business) et la prestation de services (la métropole concentrant la plupart des activités des médias du pays), alors que ceux de Brisbane cherchent la durabilité économique de leurs studios, quitte à produire de nombreux petits jeux sans grande qualité esthétique ou narrative, tandis que ceux de Melbourne mettent en avant la dimension artistique de leur démarche et la proximité avec la scène dite « indépendante ».

7 Ainsi, la géographie de la production vidéoludique est aujourd'hui prise dans les logiques de la mondialisation économique, caractérisée par la polarisation des grands studios dans certains pays, voire certaines métropoles, la nouvelle division internationale du travail, les stratégies de spécialisation de certains territoires, le soutien public à la création de jeux sur leurs territoires, mais aussi les délocalisations, la circulation des créateurs, des produits et des modèles économiques qui leur sont associés, et l'émergence de nouveaux marchés. Ces grandes tendances sont généralisables aux autres industries culturelles, mais est-ce vrai pour tous les types de jeux? Certains jeux restent fidèles à une histoire ou à des héritages proches de l'artisanat, des "beaux objets ", des savoir-faire ancrés dans les territoires (comme la marionnette lyonnaise du Guignol, en France). D'autres correspondent à des produits culturels de niche, dont la pratique reste parfois circonscrite à leurs territoires de production (comme les jeux vidéo du genre danmaku au Japon). D'autres enfin, parce qu'ils mobilisent peu ou pas d'éléments matériels (comme les jeux de mimes, de lettres, pierre-papier-ciseaux-lézard-Spock, etc), peuvent être produits ou reproduits par les joueurs au moment de leurs pratiques, à l'écart des circuits productifs et commerciaux. Mais ces différents espaces sont en interaction, Stephen Kline a par exemple montré que les conditions de production et de distribution des jeux (vidéo) influencent autant les contenus, les représentations spatiales, que les politiques commerciales des éditeurs (Kline et al., 2003).

8 Dans ces espaces de la production de jeux, nous proposons de distinguer le système productif, le système de distribution et de commercialisation et l'univers de la valorisation et de la promotion. Par « système productif ", nous entendons «l'ensemble des acteurs qui concourent à la fabrication d'un bien ou la prestation d'un service en formant un tissu de relations complexes et établissant des interdépendances », pour reprendre la définition de G. Baudelle et J. Fache $(2015,27)$. Ils analysent trois sousensembles. D'abord le système opérant qui regroupe les entreprises et acteurs qui contribuent à la production d'un bien ou d'un service ludique. Dans le cas des jeux, il s'agit des concepteurs, éditeurs, grands et petits studios. Ensuite, le système d'information qui regroupe l'ensemble des acteurs qui concourent à la diffusion et au partage d'informations et de connaissance (des outils, des marchés, des développements technologiques) nécessaires au bon fonctionnement de l'appareil productif. Ce sont les lobbies, associations et syndicats professionnels liés au secteur d'activité, mais aussi les autres organismes de production de données (chambres de commerce, chambres consulaires, IDATE ${ }^{1}$ ) et de mise en réseau. Enfin, le système de pilotage qui regroupe les acteurs qui détiennent les fonctions de commandement (acteurs publics pourvoyeurs de crédits d'impôt, clusters industriels, grands éditeurs, etc.). 
Ces systèmes productifs sont spatialisés, chaque territoire étant inégalement doté en outils de commandement et de production, et plus ou moins soutenu par la puissance publique (Kerr, 2017). Ainsi, il n'est pas possible comprendre l'émergence de Montréal comme le principal pôle mondial de l'industrie du jeu vidéo sans tenir compte d'un faisceau de facteurs localisés : la proximité avec le marché étasunien, les stratégies de délocalisation des grandes entreprises, les politiques fiscales attractives de la Province du Québec depuis la fin des années 1990, le bilinguisme qui favorise l'installation d'éditeurs francophones comme Ubisoft, mais aussi la présence de plusieurs écoles de jeux vidéo, et d'espaces intermédiaires (middleground) favorables à la créativité et à la circulation des idées (Grandadam, Cohendet, Simon, 2013). Au final, selon le rapport de 2012 de l'Alliance numérique du Québec, l'impact économique direct de l'industrie du jeu vidéo au Canada, principalement concentrée en Ontario, en Colombie Britannique et au Québec, est estimé à près de 1,7 milliard de \$. De telles analyses sont également possibles dans le cas du Japon et de l'Angleterre (Aoyama, Izushi, 2006), ou encore de la Corée du Sud. C. Paberz (2012) a ainsi mis en avant des facteurs historiques, dont le boycottage des produits technologiques japonais et des politiques publiques de soutien au développement de filières nationales, pour expliquer le positionnement actuel du pays dans le secteur des jeux vidéo, tourné vers les jeux compétitifs.

Les interactions entre les différents sous-systèmes sont aussi caractérisées par des relations de pouvoir et des rapports de force, comme J. Johns l'a montré dans le cas des studios de développement qui travaillent pour les constructeurs de consoles autorisant (ou non) la mise à disposition des jeux sur leurs machines (Johns, 2006). Par ailleurs, l'engouement récent pour le financement participatif ou crowdfounding pose la question de l'entrée des joueurs dans le système de pilotage et des limites du financement par les pouvoirs publics des industries culturelles et créatives (Brabham, 2016). La multiplication de ces interfaces brouille les catégorisations traditionnelles, comme les plateformes de financement type Kickstarter, qui concerne autant de jeux vidéo que de jeux de plateau, ou de distribution en ligne comme Steam, qui est à la fois un espace de vente (magasin en ligne), un espace de diffusion de l'information et un espace de socialisation par le jeu (forums de discussion, partage de contenus personnalisés etc.). Cette confusion est portée par des logiques aussi diverses que la dématérialisation des produits ludiques, la marchandisation de la culture, la précarité des emplois dans le secteur des industries culturelles et créatives (Dyer-Witheford, de Peuter, 2009), l'apparition d'acteurs intermédiaires (Parker, Whitson, Simon, 2017) et l'émergence de communautés de pratiques qui favorisent les échanges entre concepteurs et joueurs (Zabban, 2011).

\section{Espaces, territoires et mondes ludiques}

11 Du côté des pratiques, les différenciations spatiales mettent en tension la production de jeux avec la circulation, la réception et l'appropriation des produits par les joueurs euxmêmes. Nous proposons de distinguer ce qui se joue dans l'appropriation des espaces virtuels, c'est-à-dire les espaces dans le jeu; les territoires de la pratique, là où les joueurs jouent ; et au-delà, les mondes ludiques. 


\section{Espaces virtuels}

12 L'analyse des représentations spatiales des jeux (les espaces dans le jeu) a donné lieu à de nombreux travaux, que ce soit dans le cas des jeux vidéo (Ter Minassian, Rufat, 2008), des jeux de tablier (Borzakian, 2010) ou encore des jeux de rôle (Di Filippo, 2016). Elle offre de nombreuses pistes d'étude sur les représentations de l'espace, les ressorts politiques et idéologiques de certaines productions ludiques, mais aussi la transmission de connaissances, l'éducation par l'image et les outils numériques, les interfaces humain-machine, etc. Le jeu peut être lui-même support de représentations spatiales, inscrites dans la matérialité même de la pratique (le plateau et matériel de jeu, l'habillage graphique du boîtier, la position des joueurs dans l'espace, etc.). Ces espaces représentés dans le jeu sont des espaces virtuels, simulés dans le jeu et en interaction avec les représentations spatiales des joueurs. Ils sont composés du terrain de jeu et des plans annexes qui favorisent la lecture et l'appropriation du terrain de jeu par les joueurs. Le terrain de jeu est l'espace de la représentation, visuelle ou imaginaire, de l'action dans le jeu. Les plans de jeu correspondent à l'ensemble des éléments qui complètent le terrain de jeu et facilitent à la fois sa lecture et son appropriation par les joueurs, comme les cartes de localisation, qui sont elles-mêmes des représentations de l'espace simulé par le jeu, soit des représentations spatiales au moins au carré.

13 Le jeu peut alors tour à tour être support de discours sur l'espace, voire d'idéologies spatiales, et un outil de marketing des territoires (par exemple en utilisant un quartier ou une ville comme décor, comme dans les très nombreuses déclinaisons du Monopoly). Les travaux sur les espaces virtuels se sont surtout concentrés soit sur de l'analyse de contenu, comme les représentations du pouvoir et de la critique sociale dans le jeu vidéo Grand Theft Auto (Mauco, 2013), ou les discours revanchistes des jeux de l'oie produits à la fin du $19^{\mathrm{e}}$ siècle en France (Trémel, 2007), soit sur l'étude des interfaces de jeu, les écrans, le plus souvent dans une perspective de game design, d'ergonomie ou d'analyse des dispositifs d'interaction mécanique et analogique (Stockburger, 2006; Nitsche, 2008). Les Video Game Studies ont nourri ces approches. À cela s'ajoutent les travaux spécifiques (et plus ponctuels) de type ethnographies des mondes en ligne, qui croisent l'analyse des pratiques spatiales virtuelles et des supports de ces pratiques (Boellstorff, 2008 ; Bardzell, Odom, 2008 ; Lucas, 2013).

\section{Territoires des pratiques}

Notre deuxième entrée dans la sphère de consommation est celle des territoires des pratiques, qui regroupent les espaces dans les lesquels les personnes jouent à des jeux. Leur analyse renvoie à des interrogations fortes sur la circulation des pratiques ludiques, mais aussi la recomposition des routines du quotidien, les mobilités, la diffusion des outils et usages du numérique dans les différentes couches de la société, ainsi que la marchandisation des pratiques et les logiques commerciales qui animent la géographie des espaces ludiques. Ainsi, dans le cas des jeux dits "traditionnels» comme les échecs, le go ou le bridge, M. Borzakian rappelle les dynamiques de diffusion de leur pratique à l'échelle mondiale : " macrocéphalie est asiatique » dans la pratique du go, antagonisme historique entre blocs occidental et soviétique dans le cas des échecs, et domination persistante de l'Amérique du Nord et de l'Europe occidentale dans la pratique du bridge. Les jeux « traditionnels » donnent lieu à des appropriations spatialement différenciées en France (Borzakian, 2010, 2015). Alors que le scrabble ou le 
tarot consistent principalement en un outil de maintien de sociabilité dans les petites villes et les communes rurales, ils se prêtent à des pratiques compétitives principalement dans les centres urbains, où l'on trouve l'essentiel des meilleurs joueurs. De même, le bridge tient lieu de pratique assurant la cohésion de la grande bourgeoisie et d'individus à la trajectoire sociale ascendante dans les stations balnéaires ou les beaux quartiers des grandes villes. On y joue alors plus volontiers dans des espaces privés. Parallèlement, des compétitions nationales et internationales de haut niveau réunissent des pratiquants aux profils sociaux (un peu) plus divers, poursuivant plus clairement des objectifs comparables à ceux de sportifs professionnels ou semi-professionnels. À Haïti, on retrouve des différenciations régionales, par exemple à partir de l'analyse des jeux d'argent étudiés par M. Redon (2012). Les combats de coqs (gaguères) et les loteries (borlettes) sont tous deux des jeux de mise, mais les premières "revêtiraient une dimension plutôt rurale " quand les secondes seraient «des marqueurs de sociabilité plus urbaine» (Redon, 2012) même si, selon l'auteure, ces deux pratiques ludiques tendent aujourd'hui à se diffuser avec la progression de l'urbanisation et des réseaux de communication.

15 À une échelle plus grande, celle non plus des pays ou des régions, mais des lieux dans lesquels on joue, il est possible de dresser une géographie des pratiques ludiques. Des études récentes de type ethnographique, anthropologique ou micro-géographique portant sur les jeux vidéo ont mis en avant leur caractère situé, y compris dans une perspective historique (Triclot, 2012). À l'exception de quelques rares travaux (Geertz, 1983 ; Trémel, 2001 ; Caïra, 2007 ; Borzakian, 2010), des analyses équivalentes manquent encore pour caractériser les pratiques au-delà des jeux vidéo. Elles permettraient de montrer la diversité des espaces du jeu, les formes de socialisation qui s'y déploient, et la manière dont les pratiques ludiques contribuent à la production de territoires, et le cas échéant des identités collectives. Par exemple, dans ses travaux sur la géographie des bals et fêtes populaires en France, Dominique Crozat (2000) notait que la même activité festive et ludique pouvait être investie de significations différentes selon les territoires : dans les espaces urbains, elle serait plus anonyme et plus inclusive; dans les espaces périurbains, elle produirait une socialisation sélective dans des périmètres spatiaux bien définis; enfin dans les espaces ruraux, elle continuerait de jouer le rôle de réaffirmation d'une identité locale ancrée dans son territoire.

16 À une échelle plus fine encore, quelques travaux récents ont montré que la multiplication des supports de jeux vidéo (tablettes, smartphones, téléphones) et leur caractère nomade se sont accompagnés de la diffusion de ces pratiques dans des espaces non dédiés : désormais, on peut jouer dans les toilettes (Rufat et al. 2014), dans les transports (Ter Minassian, Boutet, 2015), au travail (Boutet, 2011), à l'université, ou encore dans les espaces publics (De Souza e Silva, 2006). A côté de ces espaces-temps du quotidien désormais investis par le jeu, il reste des espaces qui leur sont dédiés, comme les salles de jeu, les ludothèques ou les salles d'arcade. Les fonctions ludiques et de socialisation par le jeu prévalent dans ces espaces. Une hypothèse est que leur maintien, voire leur multiplication témoigne d'une recomposition des pratiques ludiques et d'une resegmentation des marchés.

17 Enfin, il convient de distinguer les espaces hybrides, à l'interface entre la sphère de production et la sphère de consommation, comme les boutiques de jeux, qui sont autant de lieux de commercialisation et de distribution des produits ludiques, mais aussi de pratiques, puisqu'il est possible d'y tester les jeux, d'y organiser des soirées à 
thème ou des tournois, mais aussi les cafés PMU en France, les centres commerciaux aux États-Unis et, dans certains pays comme l'Espagne ou le Canada, les bars et restaurants dans lesquels il existe encore des machines à sous.

\section{Mondes ludiques}

18 En dehors du jeu lui-même, les pratiques des joueurs produisent ou s'appuient sur un ensemble d'espaces, aux proximités fonctionnelles mais aux contours flous. En repartant des travaux de G. Brougère sur la « culture ludique » (2002), nous proposons de qualifier cet ensemble d'espaces de mondes ludiques. La culture ludique renvoie à la capacité à adopter une attitude propice à l'activité de jeu, aux sens et aux valeurs accordés par l'individu à l'activité ludique, et qui peuvent le conduire à l'investir selon des formes qui vont au-delà de la session de jeu : acheter des produits dérivés, se rendre à des festivals, adhérer à une association, etc. Ces différentes pratiques sociales et spatiales, qui dépassent le simple fait de jouer, sont constitutives du rapport que les joueurs entretiennent à leur pratique culturelle et brouillent la limite entre le jeu et le non-jeu, ce que V.Zabban montre par l'analyse des discussions sur les forums communautaires du jeu Age of Utopia (2011). Ainsi, les mondes ludiques correspondent à l'ensemble des espaces qui renvoient à des dynamiques sociales en lien avec le ludique, sans nécessairement être investis par l'acte de jouer en tant que tel : on y parle de jeux, on se socialise par le jeu, sans toujours pratiquer le jeu lui-même.

Ces mondes ludiques sont produits par des acteurs hétérogènes (publics et privés, grandes et petites associations, musées, festivals, conventions, etc.), en présentiel, mais aussi en ligne (forums, blogues, sites communautaires), qui génèrent des espaces qui ne sont pas des espaces de pratiques ludiques (il n'y a pas toujours des jeux de plateau ou des consoles à disposition), mais sont liées à ces cultures ludiques, qui leur donnent vie et sens pour les publics concernés, et qui contribuent à l'émergence et la structuration de communautés de pratiques. Les lieux qui les composent, qu'ils soient dédiés (un club de jeu) ou temporairement investis (un parc des expositions ou une salle de cinéma), sont le support de mobilités spatiales, de sociabilités et d'identités constitutives de communautés liées aux jeux. Les joueurs peuvent être amenés à modifier leurs pratiques ou leurs stratégies en fonction de leurs discussions avec d'autres joueurs ou de conseils recueillis sur un forum de discussion. Ensuite, les règles et représentations d'un jeu peuvent varier en fonction de l'investissement d'une communauté de joueurs prêts à créer du nouveau contenu (mods), ajouts, nouvelles variantes, niveaux ou cartes. Enfin, il arrive que les concepteurs d'un jeu recueillent l'avis des joueurs pour adapter leur produit à la demande, voire que des plateformes de distribution comme Kickstarter alternent entre production à la demande et spéculation sur la structuration d'une communauté, comme par exemple le récent jeu de plateau The 7th Continent (Ludovic Roudy, Bruno Sautter, 2017). Ces discussions sont souvent dissymétriques, et qu'elles soient ou non suivies d'effet, elles donnent parfois l'illusion d'un espace de débat pour favoriser l'implication des joueurs (Kline et al., 2003). À l'inverse, les expositions, festivals, voire la patrimonialisation et l'entrée du jeu dans les musées ont une dimension de légitimation des pratiques (Coville, 2016). Au final, ces mondes ludiques sont d'autant plus difficiles à délimiter qu'il existe aujourd'hui de nombreuses interfaces au sein de l'écosystème ludique et les lieux qui les composent sont discontinus, mais mis en étroite relation par les pratiques, les sociabilités, la circulation de l'information. 


\section{Ce que ces spatialités ont à nous dire du jeu et des joueurs}

20 Il ne s'agit pourtant pas d'un "tournant spatial» dans la recherche sur les jeux. L'ensemble de ces approches ne supplantent pas d'autres entrées qui gardent toute leur pertinence. C'est une série de coups de projecteur que les articles de ce numéro proposent sur ces différentes formes de spatialités.

L'article d'Irene Vaquinhas s'intéresse à la diffusion des jeux d'argent et de hasard dans la société portugaise $\mathrm{du} 19^{\mathrm{e}}$ et du début $\mathrm{du} 20^{\mathrm{e}}$ siècle, ainsi qu'aux tentatives des pouvoirs publics de les réguler. Elle montre les ressorts spatiaux et sociaux de cette diffusion, d'abord circonscrite aux destinations balnéaires et de villégiature des couches supérieures de la société, et l'évolution du débat public sur leur contrôle, entre condamnation, régulation et taxation, à partir du moment où les jeux d'argent gagnent les milieux populaires.

L'article de Bruno Lusso se tourne du côté de la production, en s'intéressant à la structuration du l'industrie du jeu vidéo en cluster, avec les exemples des aires métropolitaines de Lille, Lyon et Marseille. Il montre que la proximité géographique est importante pour la construction de coopérations entre les firmes, écoles, laboratoires de recherche et associations professionnelles et que l'ancrage territorial favorise également l'action volontariste des pouvoirs publics.

L'article de Marion Amalric, Brice Anselme, Nicolas Bécu, Etienne Delay, Nicolas Marilleau, Cécilia Pignon et Frédéric Rousseaux interroge l'articulation entre jeu, simulation et processus participatif autour du risque de submersion qui pèse sur la côte atlantique française, à travers l'exemple d'un «jeu sérieux» qu'ils développent, LittoSIM. Les auteurs explorent les usages en montrant que les leviers ludiques incitent les joueurs à modifier leurs pratiques professionnelles quotidiennes alors que la dimension réaliste produit un décalage qui accroit la sensibilisation aux enjeux de l'urbanisation et de l'aménagement du littoral.

24 Enfin, l'article de Frédéric Jacob et Émilie Servais s'intéresse à l'usage de jeux de géographie en contexte scolaire. Il s'appuie sur l'analyse des jeux existants à partir d'un recensement effectué dans le réseau francophone LUDUS et explore les atouts et limites d'un jeu de géolocalisation de sites d'apport volontaire de déchets, expérimenté en classe pour sensibiliser les élèves à l'aménagement urbain et aux mobilités.

Mais au-delà de ces pistes stimulantes, d'autres restent à explorer. L'articulation entre le jeu et les espaces du quotidien (où joue-t-on, à quoi et pour quoi ?) a été systématisée dans le cas des jeux vidéo (Boutet, 2012 ; Rufat et al., 2014), mais elle n'a donné lieu qu'à quelques travaux ponctuels dans le cas des autres jeux (Balli et al., 2015 ; Borzakian, 2012 ; Brougère, 1991 ; Vigne, Joncheray, 2012). Ces interrogations mériteraient d'être systématisées et étendues à l'ensemble des jeux pour chercher les éventuelles variations du sens à accorder aux pratiques de jeu, selon les espaces (domicile, transports, entreprise, formation, au musée, etc.) et selon les contextes afférents (entre amis, pour passer le temps, en formation professionnelle, etc). Elles ouvrent à leur tour sur d'autres interrogations sur la micro-géographie des pratiques ludiques et des socialisations qu'elles produisent, les espaces d'interactions et ce qu'ils révèlent des types de pratiques et des joueurs eux-mêmes. 
Plus généralement, du fait de l'organisation des acteurs industriels, des enjeux économiques et de l'attention des médias, l'entrée par la sphère de la production est plus facile dans le cas des jeux vidéo et elle a suscité moins de travaux sur les autres jeux, sauf à les rapprocher de l'industrie du jouet (Bruno, 2008) ou des industries créatives (Lusso, 2011). De même, parce qu'ils sont matérialisés sur des écrans, les espaces virtuels (dans le jeu) sont plus faciles à appréhender dans le cas des jeux vidéo. Des travaux ont bien analysé l'espace virtuel représenté pour replacer les jeux traditionnels dans leur contexte historique (Goret et al., 2009) ou culturel (Mauger, 1915), mais les analyses des spatialités dans les jeux restent des travaux ponctuels, davantage articulés avec les mondes fictionnels qu'avec les représentations spatiales (Dauphragne, 2011 ; Caïra, 2007). Dans le cas des jeux qui ne mobilisent pas de support numérique, les spatialités sont surtout étudiées sous l'angle des pratiques des joueurs à l'école (Baldner, 1984), à la bibliothèque (Devriendt, 2015), en entreprise (Balli et al., 2015), dans l'espace public (Brougère, 1991 ; Escaffre, 2005), en centre-ville (Pégard, 1998) comme en banlieue (Nunes, 2012) ou en régions (Vigne, Joncheray, 2012). Mais cela est réalisé trop souvent en vase clos, sans rattacher les pratiques aux autres espaces et territoires en jeu dans la production, la distribution et la mise en culture ludique (Borzakian, 2010). Il en résulte que c'est surtout à partir des recherches sur les jeux vidéo que les interactions entre ces différentes spatialités dans, avec et autour des jeux ont été explorées. Il nous parait donc important de souligner que ces entrées spatiales ne sont pas uniquement fécondes dans le cas des jeux vidéo. Les besoins du champ de recherche sont d'explorer plus systématiquement ces différentes spatialités, représentées dans le jeu, produites par les joueurs, ou par les systèmes territoriaux qui alimentent leurs pratiques, et surtout de les articuler, avec leurs jeux d'échelles et les représentations qu'elles mobilisent, par exemple pour interroger les fondements culturels, historiques, économiques, (géo)politiques, voire juridiques, les modèles et les circulations de ces différenciations territoriales.

\section{BIBLIOGRAPHIE}

AOYAMA Y. \& IZUSHI H. (2006), « Industry evolution and cross-sectoral skill transfers : a comparative analysis of the video game industry in Japan, the United States and the United Kingdom », Environment and Planning A, 38 (10), pp. 1843-1861.

BALDNER J.-M. (1984), « Jouer ou ne pas jouer à l'école », Espaces Temps, 25 (1), pp. 52-59.

BALLI F., AUBORT JACCARD C. (2015), » Jouer en entreprise... un gage de succès ? », Gestion, 2015, 40 (4), pp. 28-31.

BANKS J. \& CUNNINGHAM S. (2016), « Creative destruction in the Australian videogames industry », Media International Australia, 160 (1), pp. 1-13.

BARDZELL S. \& ODOM W. (2008), « The experience of embodied space in virtual worlds : an ethnography of a Second Life community », Spaces and Culture, 11 (3), pp. 239-259. 
BAUDELLE G. \& FACHE J. (2015), Les mutations des systèmes productifs en France, Rennes, Presses universitaires de Rennes.

BOELLSTORFF T. (2008), Coming of Age in Second Life : an Anthropologist Explores the Virtually Human, Princeton, Princeton University Press.

BORZAKIAN M. (2010), Géographique ludique de la France, thèse de doctorat, université ParisSorbonne.

BORZAKIAN M. (2012), « Prendre au sérieux les espaces du jeu », Géographie et cultures, (82), p. 5-9.

BORZAKIAN M. (2015), « Une géographie de la France ludique. La dimension spatiale des pratiques culturelles », Annales de géographie, 701 (1), pp. 51-75.

BOUTET M. (2011), « Un rendez-vous parmi d'autres. Ce que le jeu sur Internet nous apprend du travail contemporain », Ethnographiques.org, 23.

BOUTET M. (2012), « Jouer aux jeux vidéo avec style. Pour une ethnographie des sociabilités vidéoludiques ", Réseaux, 2012/3 ( $\left.n^{\circ} 173-174\right)$, p. 207-234.

BRABHAM D. C. (2016), « How crowdfunding discourse threatens public arts », New Media \& Society, 19 (7), pp. 1-17.

BROUGERE G. (1991) « Espace de jeu et espace public », Architecture et comportement, vol. 7, n² 2, 1991, pp. 195-176.

BROUGERE G. (2002), « L'enfant et la culture ludique », Spirale, 24 (4), pp. 25-38.

BRUNO P. (2008), « Groupes multimédias et jouets : l'exemple du groupe Disney », in G. Brougère (dir.), La ronde des jeux et des jouets, Paris, Autrement, pp. 107-117.

CAILLOIS R. (1967 [1958]), Les jeux et les hommes, Paris, Gallimard.

CAÏRA O., (2007), Jeux de rôle : les forges de la fiction, Paris, CNRS Éditions.

COVILLE M. (2016), La construction du jeu vidéo comme objet muséal, thèse de doctorat, Université Panthéon-Sorbonne.

CROZAT D. (2000), « Bals des villes et bals des champs. Villes, campagnes et périurbain en France : une approche par la géographie culturelle », Annales de géographie, 611, pp. 43-64.

DAUPHRAGNE A. (2011), «Le sens de la fiction ludique : jeu, récit et effet de monde », Strence. $n^{\circ} 2$, http://journals.openedition.org/strenae/312

DEVRIENDT J. (dir.) (2015). Jouer en bibliothèque, Villeurbanne, Presses de l'Enssib.

DE SOUZA E SILVA A. (2006), « From Cyber to Hybrid. Mobile technologies as interfaces of hybrid spaces », Space and Culture, 9 (3), pp. 261-278.

DI PHILIPPO L. (2016), Du mythe au jeu. Approche anthropo-communicationnelle du Nord. Des récits médiévaux scandinaves au MMORPG Age of Conan: Hyborian Adventures, thèse de doctorat, université de Lorraine.

DYER-WITHEFORD N., DE PEUTER G. (2009), Games of empire : global capitalism and video games, Minneapolis, University of Minnesota Press.

ESCAFFRE F. (2005), Espaces publics et pratiques ludo-sportives à Toulouse : l'émergence d'une urbanité sportive?, thèse de doctorat, université Toulouse-Jean Jaurès.

GEERTZ C., (1983 [1973]), Bali, interprétation d'une culture, Paris, Gallimard. 
GRANDADAM D., COHENDET P. \& SIMON L. (2013), « Places, spaces and the dynamics of creativity : the video game industry in Montreal », Regional Studies, 47 (10), pp. 1701-1714. GORET J.-F., TALON M., YVINEC J.-H, DERBOIS M., MOUNY S. \& POPLIN F. (2009), « Le jeu d'échecs de Noyon dans son contexte archéologique et historique », Revue archéologique de Picardie, 2009, 1 (1), pp. 79-119.

HENRIOT J. (1969), Le Jeu, Paris, Presses Universitaires de France.

HUINZINGA J. (1951 [1938]), Homo Ludens. Essai sur la fonction sociale du jeu. Paris, Gallimard.

JOHNS J. (2006), « Video games production networks: value capture, power relations and embeddedness ", Journal of Economic Geography, 6, pp. 151-180.

KERR A. (2017), Global Games : Production, Circulation and Policy in the Networked Era, New York, Routledge.

KLINE S., DYER-WITHEFORD, N. \& DE PEUTER G. (2003), Digital Play. The Interaction of Technology, Culture and Marketing, Montreal, McGill-Queen's University Press.

LUCAS J.-F. (2013), De l'immersion à l'habiter dans les mondes virtuels : le cas des villes dans Second Life, thèse de doctorat, université Rennes 2.

LUSSO B. (2011), Les dynamiques territoriales du secteur de l'image en mouvement dans les aires métropolitaines de Lille, de Lyon et de Marseille, thèse de doctorat, université Lille 1.

MAUCO O. (2013), GTA IV, l'envers du rêve américain, Paris, Questions théoriques.

MAUGER G. E. (1915). «Quelques considérations sur les jeux en Chine et leur développement synchronique avec celui de l'Empire chinois ", Bulletins et Mémoires de la Société d'anthropologie de Paris, 6 (5), pp. 238-281.

NITSCHE M. (2008), Video Game Spaces: Image, Play, and Structure in 3D Game Worlds Cambridge, The MIT Press.

NUNES J. P. S. (2012), « “Jouer son atout" : figuration et sociabilité de rue dans une banlieue de Lisbonne », Espaces et sociétés, 1, pp. 159-176.

PABERZ C. (2012), « Rendre compte d'un ancrage local. L'apport original de l'ethnologie aux Game Studies au-delà de l'ethnographie », in H. Ter Minassian, S. Rufat \& S. Coavoux (dir.), Espaces et temps des jeux vidéo, Paris, Questions théoriques, pp. 236-259.

PARKER F., WITSON J. \& SIMON B. (2017), « Megabooth: the cultural intermediation of indie games ", New Media \& Society, pp. 1-20.

PÉGARD O. (1998), « Une pratique ludique urbaine : le skateboard sur la place Vauquelin à Montréal », Cahiers internationaux de sociologie, pp. 185-202.

REDON M. (2012), « Gaguère (combat de coqs) et borlette (loterie) : quels enseignements sur Haïti ? », Géographie et cultures, n 82, p. 97-120.

RUFAT S. \& TER MINASSIAN H. (2011), Les jeux vidéo comme objet de recherche, Paris, Questions théoriques.

RUFAT S., TER MINASSIAN H. \& COAVOUX S. (2014), « Jouer aux jeux vidéo en France », L'espace géographique, 2014, 43 (4), pp. 308-323.

STOCKBURGER A. (2006), The Rendered Arena. Modalities of Space in Video and Computer Games, thèse de doctorat, London University of the Arts. 
TER MINASSIAN H. \& RUFAT S. (2008), « Et si les jeux vidéo servaient à comprendre la géographie? », Cybergéo, 418, http://journals.openedition.org/cybergeo/17502

TER MINASSIAN, H. \& BOUTET, M. (2015). «Les jeux vidéo dans les routines quotidiennes », Espace populations sociétés 2015/1-2 http://journals.openedition.org/eps/5989

TREMEL L. (2001), Jeux de rôle, jeux vidéo, multimédia : les faiseurs de mondes, Paris, Presses universitaires de France.

TREMEL L. (2007), « Jeux, éducation et socialisation politique : contribution au rappel de la permanence d'un processus », Géographie, économie, société, 9 (1), pp. 83-99.

TRICLOT M. (2012), « Jouer au laboratoire. Le jeu vidéo à l'université (1962-1979) », Réseaux, 3, pp. 177-205.

VIGNE M. \& JONCHERAY H. (2012), « La pratique des jeux traditionnels du Nord de la France », Rabaska : Revue d'ethnologie de l'Amérique française, 10, pp. 29-45.

ZABBAN V. (2011), "Ceci est un monde » Le partage des jeux en ligne : conceptions, techniques et pratiques, thèse de doctorat, université Paris-Est.

\section{NOTES}

1. Agence internationale de consultants spécialisée dans l'économie numérique.

\section{RÉSUMÉS}

La généralisation des jeux en tant que produit et pratique culturelle masque une forte hétérogénéité à la fois des outils de production, de consommation et des usages. L'objectif de cet article est de montrer en quoi l'espace et les territoires constituent une clé de lecture pertinente pour la compréhension des phénomènes ludiques dans toute leur diversité. Pour cela, nous proposons de distinguer de façon théorique d'une part la sphère de la production et la sphère de la consommation; et d'autre part les espaces virtuels, les territoires des pratiques et les mondes ludiques. Nous montrons les apports de cette proposition et identifions des besoins d'analyse qui sont autant de pistes à explorer pour des recherches futures.

The generalization of games as a product and cultural practice is covering a wide heterogeneity of production, consumption and uses. The purpose of this piece is to explain how space and territories are a reading key for the understanding of play phenomena in all their diversity. Our theoretical proposal is to distinguish the spatialities in the sphere of production and the sphere of consumption, we characterize the different spaces produced: the virtual spaces, the territories of the practices and the playful worlds. From this contribution, we present this special issue and identify the main research needs. 
INDEX

Keywords : space, territories, ecosystems, game, videogame, industry, cultural practices

Mots-clés : espace, territoire, écosystèmes, jeu, jeu vidéo, industrie, pratiques culturelles

\section{AUTEURS}

HOVIG TER MINASSIAN

Université de Tours, laboratoire CITERES

SAMUEL RUFAT

Université de Cergy-Pontoise, laboratoire MRTE

MANOUK BORZAKIAN

Laboratoire $\mathrm{ENeC}$ 